\title{
Interactions between Hippocampus and Medial Septum during Sharp Waves and Theta Oscillation in the Behaving Rat
}

\author{
George Dragoi, ${ }^{1}$ Daniel Carpi, ${ }^{1}$ Michael Recce, ${ }^{2}$ Jozsef Csicsvari, ${ }^{1}$ and György Buzsáki ${ }^{1}$ \\ ${ }^{1}$ Center for Molecular and Behavioral Neuroscience, Rutgers, The State University of New Jersey, Newark, New Jersey \\ 07102, and 2Department of Computer Science, New Jersey Institute of Technology, Newark, NJ 07102
}

The medial septal region and the hippocampus are connected reciprocally via GABAergic neurons, but the physiological role of this loop is still not well understood. In an attempt to reveal the physiological effects of the hippocamposeptal GABAergic projection, we cross-correlated hippocampal sharp wave (SPW) ripples or theta activity and extracellular units recorded in the medial septum and diagonal band of Broca (MSDB) in freely moving rats. The majority of single MSDB cells (60\%) were significantly suppressed during SPWs. Most cells inhibited during SPW (80\%) fired rhythmically and phase-locked to the negative peak of the CA1 pyramidal layer theta waves.

The septal region and the hippocampus are connected reciprocally (Raisman, 1966). The two major components of the septohippocampal projection consist of cholinergic and GABAergic cells (Shute and Lewis, 1963; Lewis et al., 1967; Köhler et al., 1984), residing in the medial septum and the diagonal band of Broca (MSDB). The cholinergic projection terminates on all types of hippocampal cells (Shute and Lewis, 1966; Frotscher and Leranth, 1985; Freund and Antal, 1988), whereas septal GABAergic neurons specifically innervate GABAergic interneurons (Freund and Antal, 1988; Gulyás et al., 1991). A subpopulation of the target GABAergic cells, in turn, projects back to the MSDB and preferentially innervates the GABAergic population (Alonso and Köhler, 1982; Gaykema et al., 1991; Tóth et al., 1993). The hippocamposeptally projecting neurons contain the calcium binding protein calbindin (Tóth and Freund, 1992; Tóth et al., 1993). Hippocampal pyramidal cells project only to the lateral septum (Alonso and Köhler, 1982; Leranth and Frotscher, 1989). The main projection of the lateral septum is subcortical, and axon collaterals to the MSDB are either absent or very sparse (Gulyás et al., 1991; Staiger and Nürnberger, 1991; Leranth et al., 1992). Therefore, the major reciprocal communication between the septum and hippocampus is mediated by the MSDB and hippocampal GABAergic cells. The functional role of this reciprocal hippocamposeptal GABAergic loop is not understood.

The hippocampal formation displays two behaviorally and physiologically distinct patterns: theta waves and sharp waves (SPWs). The importance of the septum in hippocampal theta generation has been documented by numerous experiments. In an

Received March 8, 1999; revised May 3, 1999; accepted May 5, 1999.

This work was supported by National Institutes of Health Grants NS34994 and MH54671. We thank Z. Borhegyi, H. Hirase, C. King, and Z. Nadásdy for help and support and T. F. Freund for his comments on this manuscript.

Correspondence should be addressed to György Buzsáki, Center for Molecular and Behavioral Neuroscience, Rutgers University, 197 University Avenue, Newark, NJ 07102.

Copyright (C) 1999 Society for Neuroscience $\quad 0270-6474 / 99 / 196191-09 \$ 05.00 / 0$
Because both SPW and the negative peak of local theta waves correspond to the maximum discharge probability of CA1 pyramidal cells and interneuron classes, the findings indicate that the activity of medial septal neurons can be negatively (during SPW) or positively (during theta waves) correlated with the activity of hippocampal interneurons. We hypothesize that the functional coupling between medial septal neurons and hippocampal interneurons varies in a state-dependent manner.

Key words: EEG; GABAergic neurons; interneurons; ripples; cholinergic system; lateral septum

early formulation, neurons in the MSDB have been assumed to play the role of a pacemaker by providing a coherent and rhythmic drive to the hippocampus (Green and Arduini, 1954; Petsche et al., 1962; Stumpf et al., 1962; Winson, 1978; Andersen et al., 1979). Subsequent studies have shown that MSDB GABAergic cells have the intrinsic propensity to oscillate at theta frequency (Serafin et al., 1996) and suggested that a coordinated output of the GABAergic and cholinergic populations is responsible for phase-locking hippocampal neurons (Stewart and Fox, 1989; Lee et al., 1994; Brazhnik and Fox, 1997). In contrast, other studies could not confirm the hypothesis that either the putative cholinergic or GABAergic MSDB neurons discharge coherently during the theta cycle (Gaztelu and Buño, 1982; Stewart and Fox, 1989, 1990; King et al., 1998). Two major technical problems have hampered progress in this area of research. The first is the lack of unambiguous criteria for the identification of neuronal types from the extracellularly recorded spike patterns and the lack of histological identification of intracellularly studied neurons in acute experiments. The second problem is to isolate the contribution of the participating members in multiple loops (Vertes and Kocsis, 1997).

Theta rhythm is generated by a consortium of various pathways and mechanisms. In contrast, SPW bursts are known to emerge in the recurrent collateral system of CA3 pyramidal cells (Buzsáki et al., 1983). The synchronous discharge of pyramidal cells induces high frequency and coherent discharge of the target interneurons, including the calbindin-immunoreactive subclass, with axonal projection to the MSDB (Tóth and Freund, 1992). We reasoned, therefore, that by studying the correlation between hippocampal SPWs and MSDB neuronal activity, we can assess the unidirectional effect of hippocampal interneurons on MSDB neuronal populations. In addition, previous research has established that both pyramidal neurons and various classes of interneurons display their highest discharge probability around the negative peak of theta waves in the CA1 pyramidal layer (Fox et al., 1986; 
Skaggs et al., 1996; Csicsvari et al., 1999). Using both SPWs and theta oscillations as reference signals for the maximum discharge probability of interneurons, we examined their impact on the activity of MSDB neurons in these two respective states.

\section{MATERIALS AND METHODS}

Animals and surgery. Ten adult male Sprague Dawley rats (weighting $300-500 \mathrm{gm})$ were anesthetized with a mixture $(4 \mathrm{ml} / \mathrm{kg})$ of ketamine $(25$ $\mathrm{mg} / \mathrm{ml})$, xylazine $(1.3 \mathrm{mg} / \mathrm{ml})$, and acepromazine $(0.25 \mathrm{mg} / \mathrm{ml})$ administered intramuscularly, following National Institutes of Health guidelines. Tungsten high-impedance electrodes (0.5-1 M $\Omega$; Micro Probe Inc., MD) were mounted on movable drives and used to record the unit activity in the MSDB area. Each complete turn of the microdrive moved the electrode $0.3 \mathrm{~mm}$ axially. A hole $(1 \mathrm{~mm}$ in diameter $)$ was drilled above the septal area in the midline. After cutting the dura mater, the sagittal sinus was gently moved aside, and the tungsten electrode was lowered into the midline, $\sim 1 \mathrm{~mm}$ above the medial septum [anteroposterior (AP), $0-0.7$; lateral (L), 0; ventral (V), 5], according to the atlas coordinates of Paxinos and Watson (1997). In one rat, two such microdrives were used, one for recording from the MSDB and the other one from the lateral septum. The hippocampal recording electrodes were constructed from $50-\mu \mathrm{m}$-diameter stainless steel wire coated with insulation (California Fine Wire, Grover Beach, CA). Two of these wires, with $250 \mu \mathrm{m}$ vertical and $300 \mu \mathrm{m}$ horizontal distance between their tips, were mounted on the same type of microdrive as in the high-impedance electrode. These electrodes were unilaterally implanted through holes drilled above the dorsal hippocampus (AP, $4 ; \mathrm{L}, 2.5 ; \mathrm{V}, 1.5$ ). In addition, a pair of stainless steel wires $(100 \mu \mathrm{m}$ in diameter) with $0.5 \mathrm{~mm}$ vertical tip separation was placed in the fimbria $(\mathrm{AP},-1.3 ; \mathrm{L}, 1 ; \mathrm{V}, 3.8)$ to stimulate the commissural afferents to the CA1 region. The microdrives, as well as the stimulating electrodes, were fixed to the skull with dental acrylic. Two stainless steel screws driven into the bone above the cerebellum served as reference and ground electrodes. Two additional screws in the frontal bone acted as anchors. After the operation, the rats were kept one to a cage, weighed, and inspected daily. Recording began after 1 week of recovery.

Recording and data analysis. The wire electrodes were lowered into the hippocampus until the CA1 pyramidal layer was identified. Positioning of the hippocampal electrodes was aided by the response evoked by commissural stimulation and by the presence of field ripples $(200 \mathrm{~Hz}$ oscillations) associated with unit activity (Ylinen et al., 1995). After stable recording from the pyramidal cell layer, the tungsten electrode was moved into the MSDB until discriminable septal units were recorded. Recordings were performed in the home cage while the rat was sleeping. Rapid eye movement sleep (REM) was distinguished from slow-wave sleep (SWS) by the presence of continuous theta waves and immobile sleeping posture. The duration of recording sessions varied between 5 and $55 \mathrm{~min}$. The electrical signals were amplified, bandpass filtered $(1 \mathrm{~Hz}$ to $5 \mathrm{kHz}$; model 12-64 channels; Grass Instruments, Quincy, MA), digitized with 12-bit resolution at 10 or $20 \mathrm{kHz}$ (ISC-16 analog-to-digital converter; RC Electronics, Santa Barbara, CA), and recorded continuously using a 486 personal computer. The data were stored on $4 \mathrm{~mm}$ digital audio tapes and analyzed off-line. To detect the unit activity, the wide-band recorded signals were digitally high-pass filtered $(800 \mathrm{~Hz}-5$ $\mathrm{kHz}$ ). Septal and hippocampal units were identified and isolated from the extracted spikes based on amplitudes and wave shapes using a manual cluster cutting method, as described recently (Csicsvari et al., 1998). Units showing a refractory period in the autocorrelogram larger than 2 msec were further considered as single units. The beginning, middle, and end of the hippocampal ripple episodes were detected by applying an amplitude threshold to the previously bandpass $(150-250 \mathrm{~Hz})$ filtered hippocampal EEG data. Hippocampal theta epochs were automatically identified by using the theta/delta power ratio, whereas the individual theta cycles were detected after bandpass $(5-28 \mathrm{~Hz})$ filtering the recorded data (Csicsvari et al., 1998). For analysis, 15-600 sec of theta epochs and 100-1500 successive SPW events were used. The middle of the ripples and the negative peak of the theta waves were subsequently used as zero reference points to compute unit-to-ripple crosscorrelograms and unit-to-theta phase histograms (Csicsvari et al., 1999). Autocorrelation and cross-correlation functions were calculated separately during theta waves, sharp waves, and between sharp wave bursts. Because the number of action potentials used for the construction of autocorrelograms and cross-correlograms varied from cell to cell, the histograms were normalized by dividing each bin by the number of

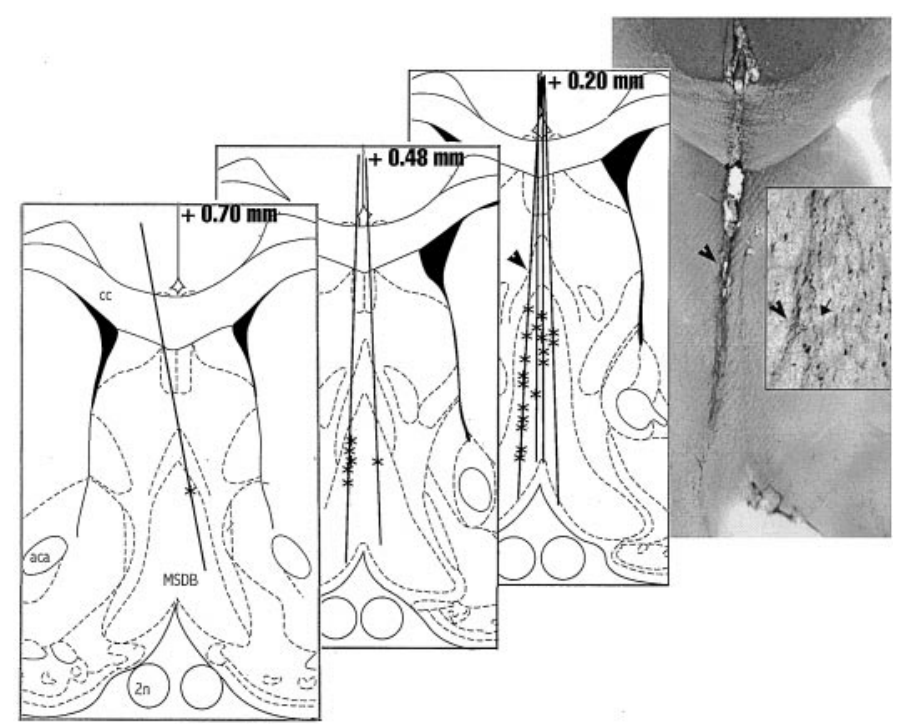

Figure 1. Location of the recorded units in MSDB. Lines indicate electrode tracks. Asterisks, Estimated location of recording sites. Image, ChAT immunostaining illustrating the recording track. Inset, Parvalbumin immunostaining of an adjacent section; the arrow points to the midline. Arrowheads mark the tracks of the same electrode on diagram and histological sections. Numbers represent distance (in millimeters) from bregma. aca, Anterior commissure; $c c$, corpus callosum; $2 n$, optic nerve. Anatomical diagrams adapted from Paxinos and Watson (1997).

reference events. Thus, the histograms reflect discharge probabilities. The method for constructing phase histograms have been described previously (Csicsvari et al., 1999). In short, each spike was assigned to a given phase (bin size of $20^{\circ}$ ) of the normalized field cycle (ripple-theta). To reduce bin-border variability, the action potential times were substituted with a Gaussian kernel function. Histograms with mean bin value of less than five spikes were excluded from the analysis. Theta and ripple averages were obtained by averaging filtered signals, using the positive peaks of the ripples waves and the negative peaks of the theta cycle as the zero reference.

Histology. After the completion of the experiments, the rats were deeply anesthetized with a high dose of Nembutal $(100 \mathrm{mg} / \mathrm{kg}$, i.p.). The electrodes remained in situ, and the rats were perfused transcardially with saline, followed by a phosphate-buffered (PB) $(0.1 \mathrm{M})$ fixative $(4 \%$ paraformaldehyde, $0.15 \%$ picric acid, and $0.05 \%$ glutaraldehyde). The brains were removed from the skull, blocks of the MSDB and hippocampus were dissected, and $80-\mu \mathrm{m}$-thick sections were cut on a vibratome. The brains of five rats were stained with the Nissl method, and the remaining five were processed for parvalbumin or choline acetyltransferase (ChAT) immunostaining on alternate sections, as follows. After washing, the brain slices were treated with $0.5 \%$ Triton X-100 diluted in $0.1 \mathrm{M} \mathrm{PB}, \mathrm{pH}$ 7.4. Next, the sections were incubated overnight in the primary antisera, rabbit anti-parvalbumin $(1: 1500)$ or rat anti-ChAT (1:10; Boehringer Mannheim, Mannheim, Germany), followed by the secondary antibodies (biotinylated anti-rabbit IgG; 1:200; Vector Laboratories, Burlingame, $\mathrm{CA}$ ) and anti-rat Ig-biotin $\mathrm{F}\left(\mathrm{ab}^{\prime}\right)_{2}$ fragment (1:200; Boehringer Mannheim) for $2 \mathrm{hr}$, followed by avidin-biotin-horseradish peroxidase complex (1.5 hr; 1:200; Vector Laboratories). The immunoperoxidase reaction was developed using 3,3'-diaminobenzidine (1:200; Sigma, St. Louis, MO). The brain sections were dried on gelatin-coated slides, dehydrated, and covered with Depex for light microscopy. A single septal track was made per animal, and it was recovered in all animals. Only rats with tracks that undoubtedly penetrated parvalbumin- and/or ChAT-rich areas within the borders of the MS/DB were considered for further analysis.

\section{RESULTS}

In 7 of the 10 operated rats, the recording electrode passed through the MSDB, as verified by the histological analysis (Fig. 1). Only data from these rats are included in this report. The position of the recorded unit was estimated by histological veri- 
HIPPOCAMPAL FIELD

A

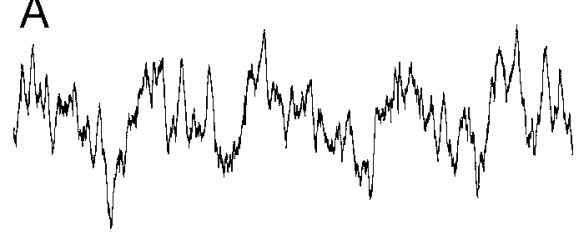

$250 \mu \mathrm{V}$
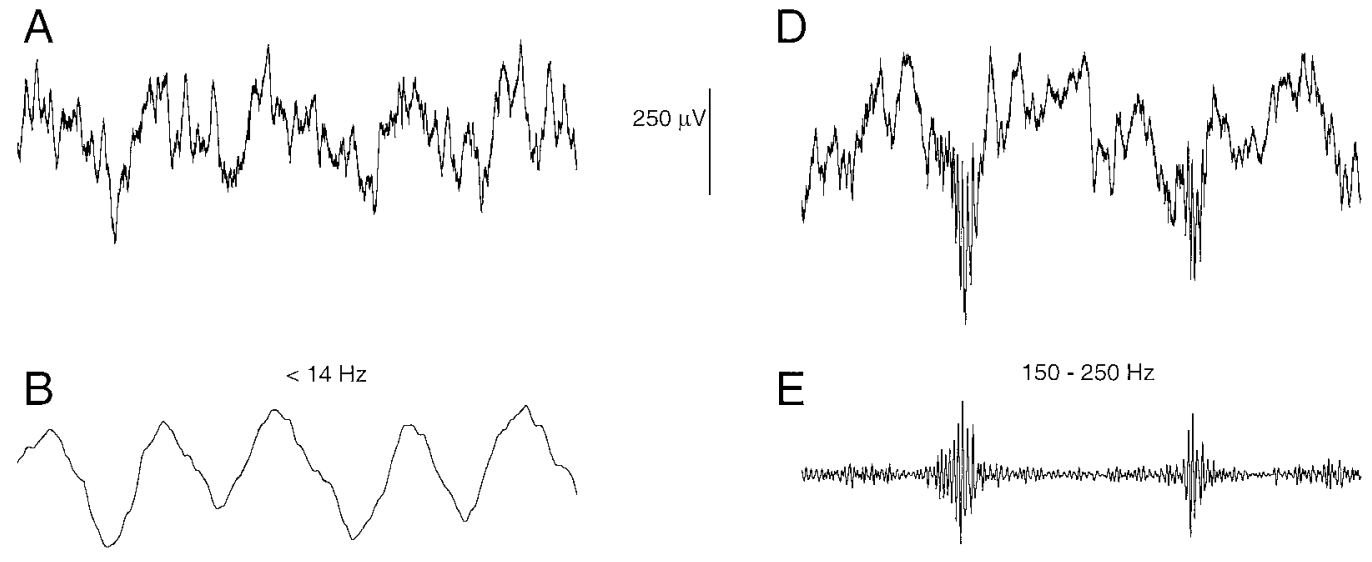

MSBD UNIT
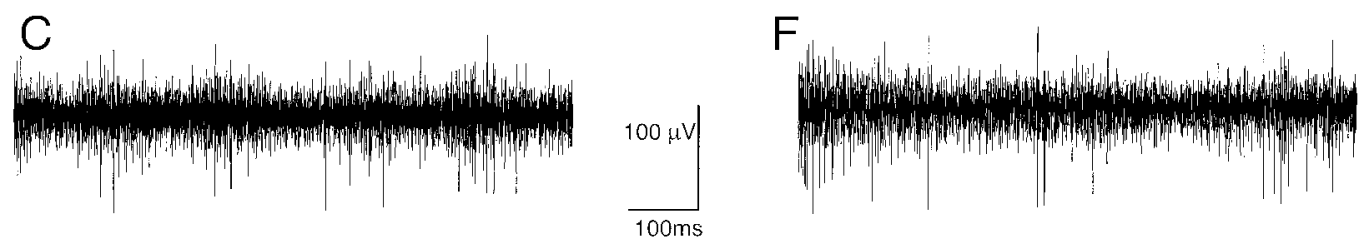

Figure 2. Hippocampal field and septal unit activity during theta waves $(A-C)$ and SPW ripples $(D-F)$. $A, D$, Wide-band $(1 \mathrm{~Hz}$ to $5 \mathrm{kHz})$ recording of a theta oscillation $(A)$ and ripples $(D)$ from CA1 pyramidal layer. $B$, Filtered derivative (14 $\mathrm{Hz}$ low-pass) of $A$. $E$, Filtered derivative $(150-250 \mathrm{~Hz})$ of $D$. $C, F$, Filtered MSDB unit activity $(0.8-5 \mathrm{kHz})$ recorded simultaneously with the hippocampal traces. Note rhythmic multiple-unit discharge in MSDB during theta. Note also the apparent lack of the MSDB unit activity during hippocampal ripples.

fication of the tip of the electrode and the number of turns of the microdrive. A total of 25 putative single MSDB units that fired during both REM and SWS were analyzed further. From each recording site, only the most well isolated single unit was processed (Fig. 2). Although the refractory test assured us that the units included in the analysis were derived from a single cell, smaller amplitude units, generated by the same neuron, might have been lost. Although this omission error does not affect the main conclusion of our findings, it may affect the firing frequencies reported below.

\section{Discharge patterns of MSDB cells during theta oscillation}

Because nearly all available information regarding the firing patterns of MSDB neurons has been collected during hippocampal theta activity, we first examined the phase and amplitude modulation of the recorded cells during the theta cycle. The cells were divided into three major groups according to previously established criteria (Gaztelu and Buño, 1982; Alonso et al., 1987; King et al., 1998). All type 1 cells $(n=18)$ showed rhythmicity in their autocorrelograms and fired phase-locked to hippocampal theta. Type 1A subgroup ( $n=2$; firing rates, 90.1 and $48.8 \mathrm{~Hz}$; peak intraburst firing rates, 500.5 and $416.6 \mathrm{~Hz}$, respectively) emitted more than five spikes during each theta cycle. Theta-associated repeating bursts were revealed as a small secondary peak at 85-95 msec in the interspike (ISI) histogram. Type 1B cells $(n=6$; mean firing rate, $21.5 \pm 7.4 \mathrm{~Hz}$; peak intraburst frequency, $275.6 \pm 66.7 \mathrm{~Hz}$ ) fired shorter bursts (two to four spikes) per theta wave reflected as a secondary peak at $100-125 \mathrm{msec}$ in the ISI histogram. Type $1 \mathrm{C}$ neurons $(n=10$; firing rate, $20.5 \pm 13.8 \mathrm{~Hz})$ did not show a clear bursting behavior, but their ISI histograms occasionally revealed theta-associated rhythmicity. Type 2 cells
( $n=5$; firing rate, $16.28 \pm 8.5 \mathrm{~Hz}$ ) showed no rhythmicity in their autocorrelograms but fired phase-locked to the hippocampal theta. Type 3 cells ( $n=2$; firing rates, 2.3 and $2.7 \mathrm{~Hz}$ ) showed no theta-related rhythmicity and were not phase-locked to the theta cycle.

\section{Activity of MSDB neurons during SPW ripples}

SPWs in the CA1 stratum radiatum are associated with synchronous cell discharge of both pyramidal cells and interneurons. Concurrent with the SPW, a fast-field oscillation (200 Hz ripple) is present in the pyramidal layer (Buzsáki et al., 1992). Using the ripples as a reference event, peri-ripple unit firing probability histograms were constructed. The histograms were normalized by dividing the cumulative bin events by the number of ripples. At least 100 SPW events and 3000 spikes were used for the construction of a histogram.

The comparison between hippocampal ripples and discharge of MSDB neurons revealed that none of the putative single units increased its firing frequency during SPW ripples. On the other hand, the discharge probability of many of the recorded units decreased selectively during SPW ripples. The SPW concurrent suppression of neuronal discharge in a representative type 1 MSDB neuron is shown in Figure $3 E$. The neuron displayed highly rhythmic activity during hippocampal theta activity, as shown by the theta-unit phase histogram and its autocorrelation function and ISI histogram (Fig. $3 A-C$ ). During SPW ripples, the multiple-unit activity recorded from the CA1 pyramidal layer increased several-fold (Fig. 3D). Concurrent with the increased activity of hippocampal neurons, the discharge probability of the MSDB unit decreased substantially (Fig. $3 E$ ).

To quantify this relationship between hippocampal SPW ripples and MSDB unit activity, the firing probability around the 


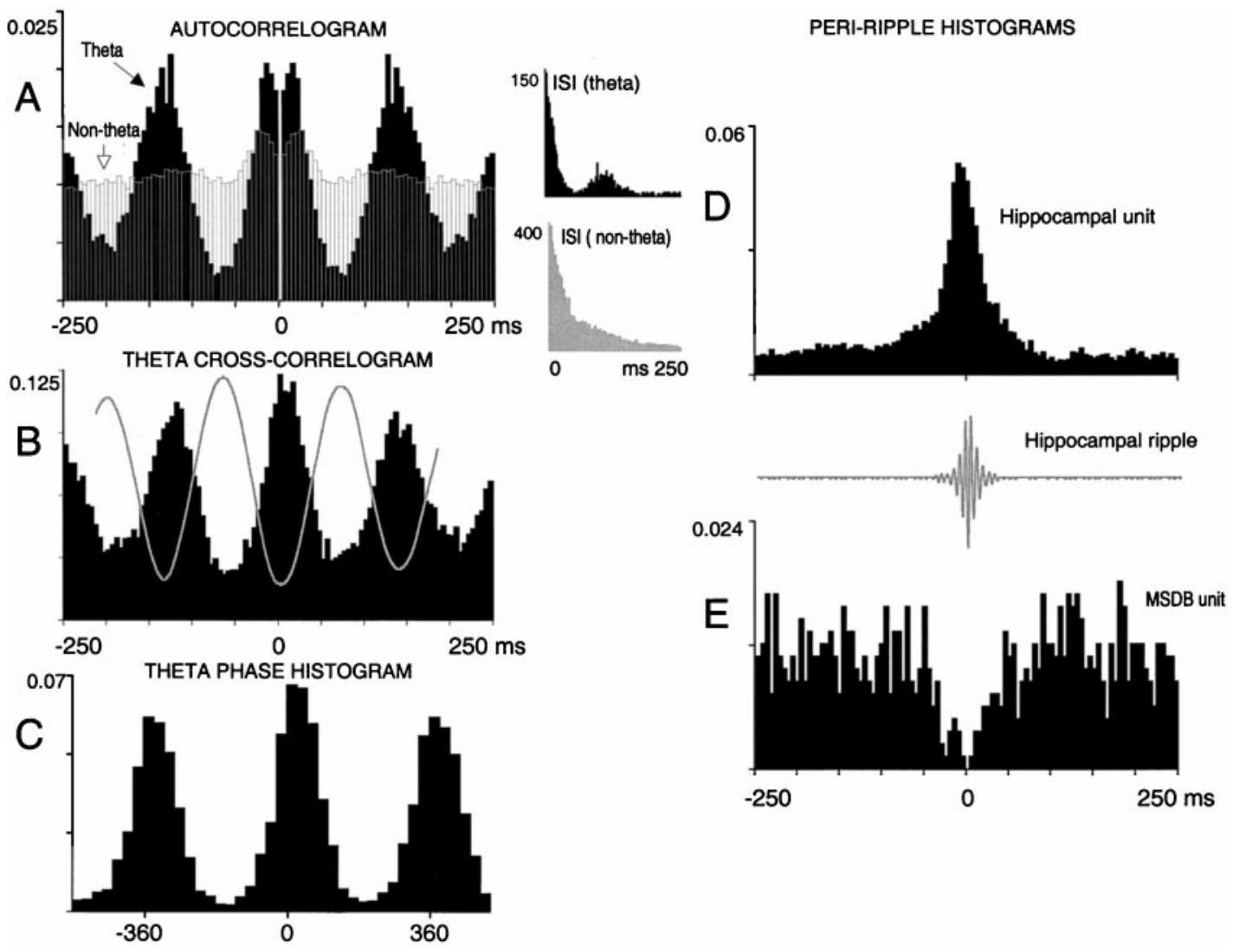

Figure 3. Discharge correlates of a rhythmically bursting MSDB single unit (type 1B) during hippocampal theta, nontheta, and SPWs. Nontheta periods included events during and in between SPWs. A, Autocorrelogram of the MSDB unit during hippocampal theta (black) and nontheta (gray) epochs. Insets, ISI histograms during theta and nontheta. Note high degree of rhythmicity during theta. $B$, Cross-correlogram between MSDB unit and negative peaks of theta waves and averaged theta field (gray line). $C$, Theta phase histogram of the MSDB single unit. Note that the MSDB unit was phase-locked to the negative peak of theta, recorded from the CA1 pyramidal layer. $D, E$, Cross-correlogram between hippocampal ripple (gray line) and locally recorded multiple-unit activity $(D)$ and MSDB unit $(E)$. Note decreased MSDB discharge during the ripple. Values on the $y$-axes represent firing probabilities (except for ISIs).

ripple peak ( $\pm 50 \mathrm{msec}$ from the peak) was compared with the baseline discharge of the putative single neurons. Baseline discharge was calculated from the firing rate in the intervals between -250 to $-75 \mathrm{msec}$ and 75 to $250 \mathrm{msec}$. The firing rate of a given neuron was arbitrarily judged as suppressed when the value of at least three neighboring $5 \mathrm{msec}$ bins around the ripple peak was more than two SDs below the baseline probability. Fifteen of the 25 putative single cells were significantly inhibited during the occurrence of hippocampal SPWs (Fig. 4). For comparison, the activity of the MSDB neurons is displayed together with SPWrelated discharge of pyramidal cells and hippocampal interneurons from a previous study, recorded under identical conditions (Fig. 4A,B) (Csicsvari et al., 1999). The length of significant discharge suppression varied from three $5 \mathrm{msec}$ bins (our criteria) to 11 consecutive bins ( $55 \mathrm{msec})$. Of the inhibited cells, 14 were type $1(93.3 \%), 1$ belonged to the type 2 category $(6.7 \%)$, and none was from the type 3 group $(0 \%)$. The distribution of significantly suppressed cells in the various cell groups, rhythmic (type 1) versus nonrhythmic (types 2 and 3), differed significantly ( $p<0.005 ; \chi^{2}$ test of independence). The remaining cells did not show significant suppression according to our criteria. Nevertheless, the grand average of the cross-correlograms of these neurons also showed some suppression of neuronal firing at around the center of the SPW burst.

Two of the 15 SPW-suppressed neurons, showing the best rhythmic theta modulation (type $1 \mathrm{~A}$ ) (Figs. 5E, F, 6E), continued to display theta rhythmicity during slow-wave sleep (Fig. $5 B, C$ ) (Barrenechea et al., 1995; Brazhnik and Fox, 1997). One of them was recorded simultaneously with a unit from the lateral septum (Fig. 5D) monitored through a second electrode. Whereas the lateral septal unit showed a clear SPW ripple-associated increase in its discharge pattern (Fig. 5A) (Carpi et al., 1997), the medial septal unit continued to display rhythmicity, as judged by its autocorrelogram (Fig. 5C). The burst-repeating frequency, however, was somewhat faster in the absence of theta $(110 \mathrm{msec})$ than during theta $(140 \mathrm{msec})$.

\section{Population phase-locking of MSDB neurons to the theta cycle}

Cross-correlation between MSDB unit activity and hippocampal theta waves, derived from the CA1 pyramidal layer, revealed individual variability in the preferred theta phase of units (compare Figs. $3 B, C, 5 E$ ). Although the chemical nature or connectivity of the recorded MSDB neurons could not be revealed in the present study, we used the SPW-induced suppression of firing rate as an independent measure for grouping MSDB neurons. As Figure $6 C$ shows, MSDB neurons, which were significantly suppressed during SPW (Fig. $4 C$ ), were phase-locked to the negative part of the theta waves recorded from the CA1 pyramidal layer. Thus, this subgroup of neurons showed a phase-coherent popu- 

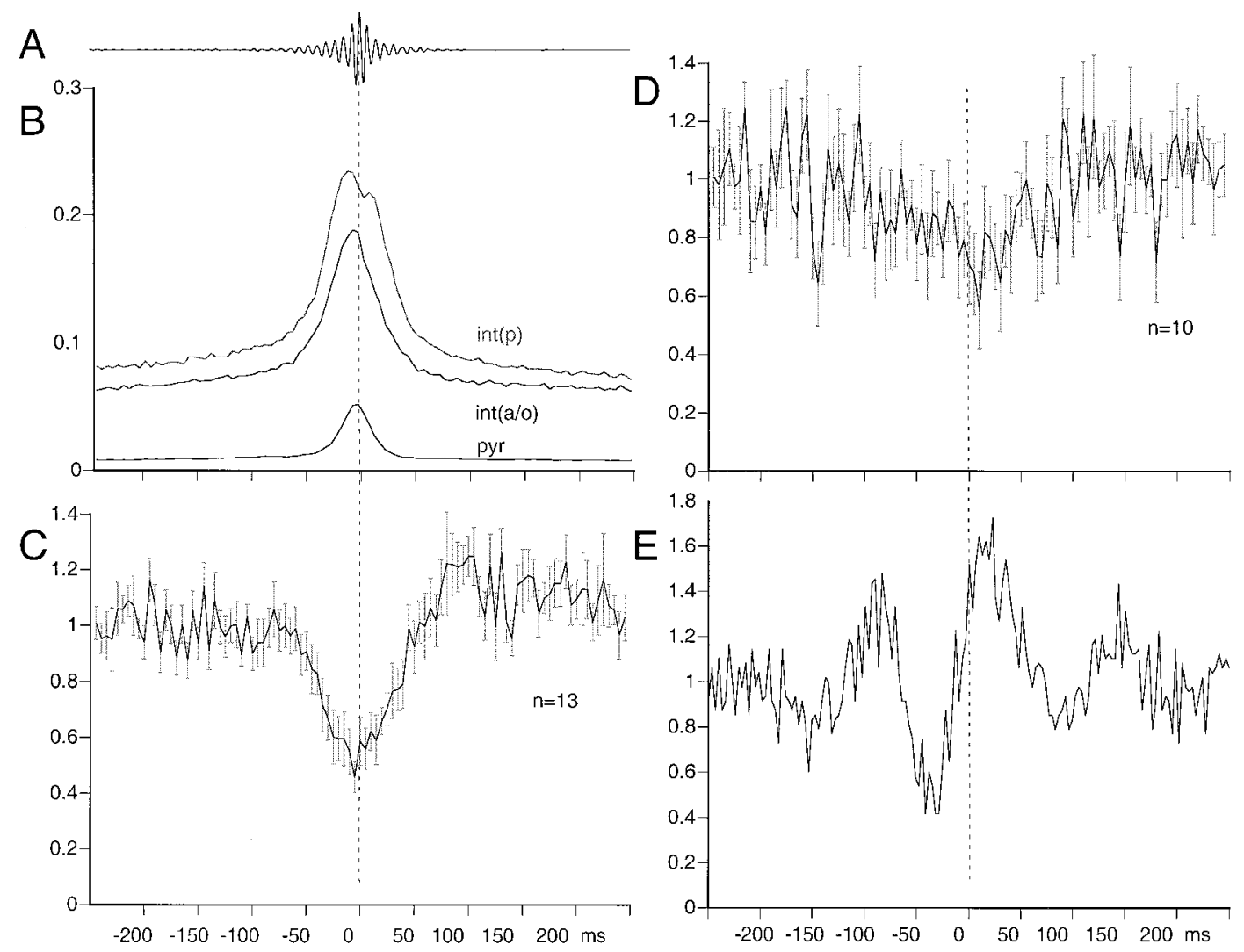

Figure 4. Firing rates of MSDB neurons are suppressed during hippocampal SPWs. $A$, Average field ripple wave. $B-E$, Discharge probabilities of hippocampal and MSDB neurons during hippocampal SPW-associated ripples. $B$, Average firing probability of different neuronal subgroups recorded from the CA1 area of the hippocampus. Note almost threefold increase in discharge probability of the interneurons in the alveus/stratum oriens and in the pyramidal layer during ripples (data replicated from Csicsvari et al., 1999). $C$, Average peri-ripple firing probability histogram for MSDB cells, which showed a significant reduction of discharge probability during SPW ripples $(n=13)$ (see Materials and Methods). Type 1A neurons are not included $(n=2)$. $D$, Peri-ripple firing probability histogram for MSDB cells, which failed to show a significant ripple-related reduction of discharge probability $(n=10)$. Error bars indicate SE. E, Peri-ripple firing probability histogram of a type 1A cell. Note the early inhibition and the maintenance of a theta-like oscillation during ripple-centered epochs. Peak of the hippocampal ripple, $0 \mathrm{msec}$. Histograms in $C-E$ were normalized to the baseline firing probability calculated from -250 to $-150 \mathrm{msec}$. $\operatorname{int}(p)$, Interneurons recorded from the pyramidal layer; int $(a / o)$, Interneurons from the alveus and stratum oriens; pyr, CA1 pyramidal neurons.

lation synchrony relative to the theta cycle. The maximum probability of their discharge corresponded to the maximum discharge of interneurons located in both the pyramidal layer and alveus/stratum oriens (Fig. 6A,B; Csicsvari et al., 1999). Thus, in contrast to what is observed during SPW, hippocampal interneurons and a subgroup of MSDB neurons discharge synchronously during theta. The discharge relationship of the two type $1 \mathrm{~A}$ neurons to theta waves are shown separately (Fig. 6E). These were the only SPW-suppressed MSDB neurons whose discharge probability coincided with the positive portion of the CA1 pyramidal layer theta waves. The remaining 10 neurons discharged (SPW-unrelated) at a maximum rate on the positive-going phase of the theta cycle (Fig. 6D).

\section{DISCUSSION}

The observations of the present experiment are that (1) a concerted discharge of hippocampal neurons during the SPW burst is associated with a concurrent suppression of discharge activity of most MSDB neurons, and (2) the maximum discharge of the SPW-inhibited MSDB neurons corresponded to the maximum discharge of CA1 interneurons during theta activity. These findings indicate that the physiological relationship between popula- tions of MSDB and hippocampal neurons varies as a function of behavior.

\section{Suppression of MSDB neurons during hippocampal SPWs}

Because SPWs are generated by the intrinsic circuitry of the hippocampus, correlation of MSDB neurons with this physiological pattern allowed for the direct investigation of the hippocampal influence on the MSDB neurons. Most MSDB neurons were suppressed during SPW ripples and none of the cells showed SPW-associated discharge in their firing probability. Although it is likely that both cholinergic and GABAergic neurons were sampled by our recordings, the anatomical identity of our cells remained undisclosed.

The hippocampal projection to MSDB derives from GABAergic interneurons of the CA3 and CA1 regions (Alonso and Köhler, 1982; Gaykema et al., 1991; Tóth and Freund, 1992; Tóth et al., 1993). The hippocampofugal projection terminates mostly on the GABAergic population of the MSDB, but synapses on cholinergic cells were also noted (Tóth et al., 1993). The intraMSDB synaptic organization among the various neuronal types is not known, but it has been assumed that MSDB GABAergic cells 

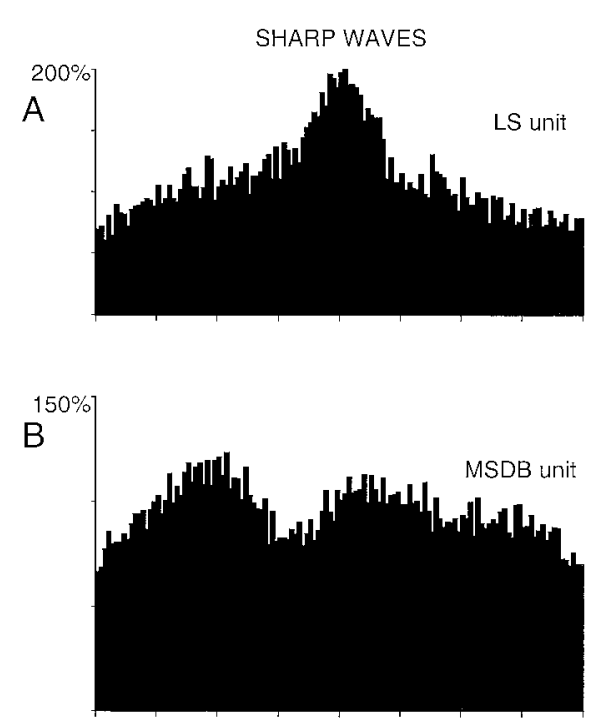

MSDB UNIT AUTOCORRELOGRAMS

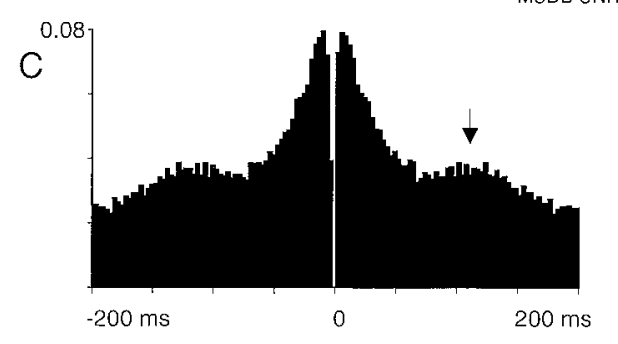

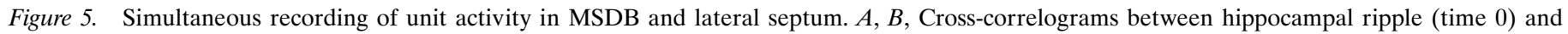

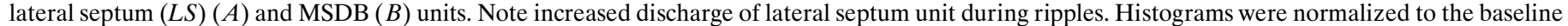

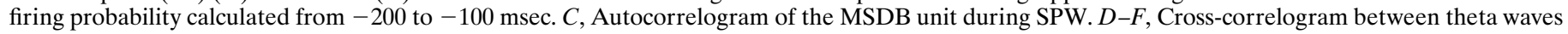

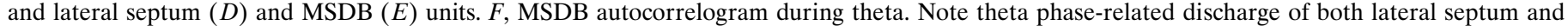

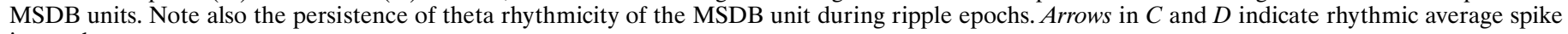
intervals.

give off local collaterals and innervate neighboring cholinergic cells (Leranth and Frotscher, 1989; Van der Zee and Luiten, 1994). In light of these anatomical observations, one would expect that SPW-mediated suppression of MSDB neurons should relieve the inhibition these neurons may exert on cholinergic neurons. If this were the case, one may expect to find that at least some MSDB neurons increased their discharge rate during SPW. This was not found to be the case, however. Our physiological observations, therefore, suggest that intraseptal GABAergic innervation of other types of MSDB cells is considerably weaker than the concerted effect of hippocampal GABAergic inhibition of MSDB neurons. It should be noted that the activity of a subgroup of hippocampal interneurons ("anti-SPW" cells) (Csicsvari et al., 1999) are also suppressed during SPWs. It remains to be seen whether hippocampal anti-SPW and MSDB neurons are innervated by the same GABAergic neurons.

What is the physiological function of the reciprocal inhibitory innervation between the hippocampus and MSDB during SPWs? Because SPWs are thought to be initiated by the recurrent collateral system of the hippocampal CA3 region, suppression of MSDB neurons is likely brought about by the increased activity of septally projecting GABAergic hippocampal interneurons. In return, the decreased activity of the target MSDB cells may have important consequences on the activity of hippocampal neurons, as well. Decreased firing of MSDB GABAergic neurons may contribute to the termination of SPW burst by disinhibiting hip- pocampal interneurons (Tóth et al., 1997), suggesting a regulatory negative feedback role for the hippocampus-MSDB-hippocampus loop. Indeed, after fimbria-fornix lesion, SPWs are converted into large interictal epileptic-like spikes associated with hypersynchronous activity of pyramidal cells (Buzsáki et al., 1989). Furthermore, decreased activity of cholinergic neurons may affect backpropagation of action potentials in hippocampal pyramidal cells (Tsubokawa and Ross, 1997) and influence synaptic plasticity (Magee and Johnston, 1997; Markram et al., 1997).

The SPW-associated activity of MSDB neurons may also exert an important effect on the hippocampo-lateral septum-hypothalamus pathway. GABAergic neurons in the MSDB are believed to innervate the GABAergic neurons in the lateral septum (Swanson and Cowan, 1979; Staiger and Nürnberger, 1989; Kiss and Patel, 1991). Decreased inhibition of the lateral septal cells during SPWs therefore may provide temporal windows for the hippocampal output to influence hypothalamic targets. The increased discharge of lateral septal neurons during SPWs is consistent with this assumption (Carpi et al., 1997).

\section{Contribution of MSDB neurons to the theta rhythm}

In agreement with previous studies, we found that neurons in the MSDB form several functional subgroups: rhythmic neurons phase-locked to theta, nonrhythmic but phase-locked cells, and a smaller group of cells that were neither rhythmic nor phase- 


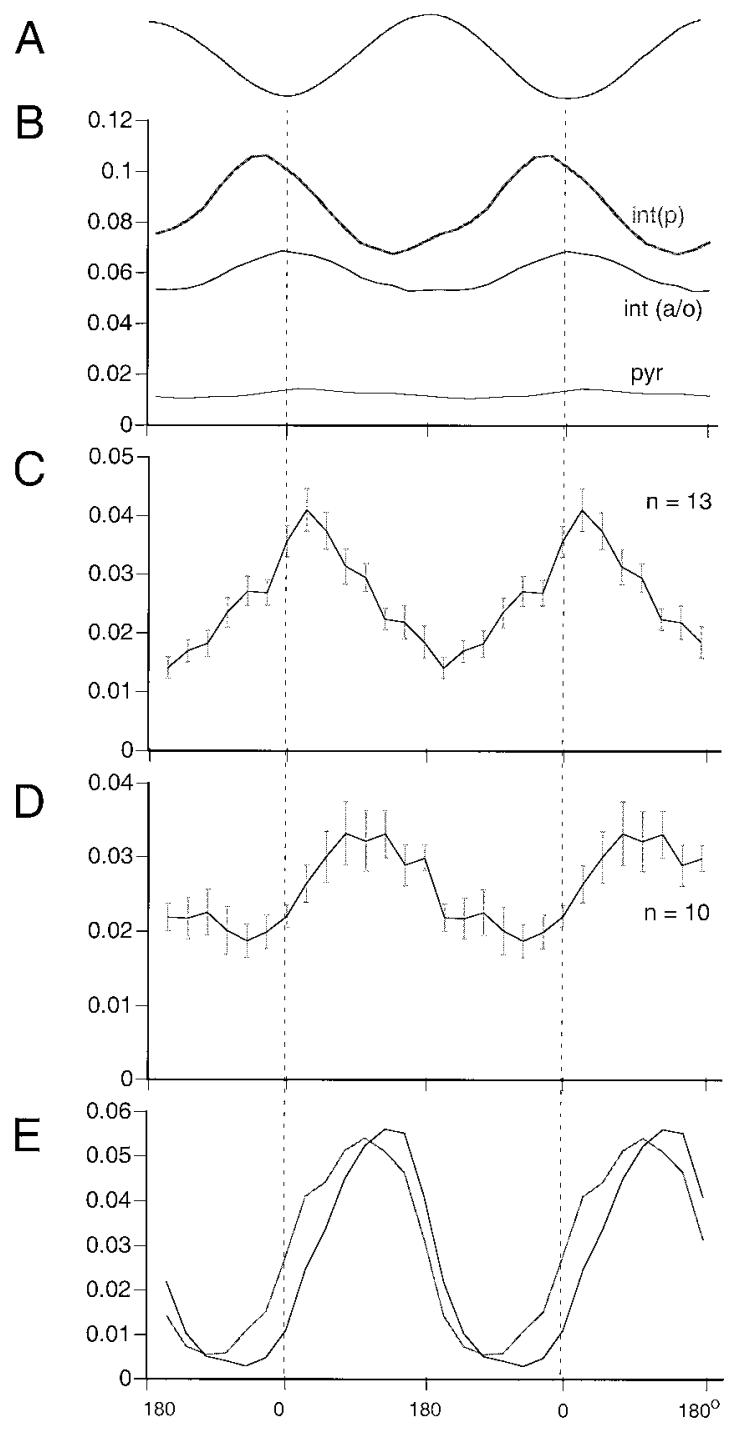

Figure 6. Average phase distribution of hippocampal and MSDB units in relation to the CA1 pyramidal layer theta. $A$, Average theta field wave. Two theta cycles are shown to facilitate comparison. Dashed vertical lines mark the negative peak of hippocampal theta. $B$, Average phase distribution of different CA1 cell populations. Note maximum discharge probability of pyramidal layer $[\operatorname{int}(p)]$ interneurons and alveus/stratum oriens $[\operatorname{int}(a / o)]$ interneurons just before and at the peak of the local theta, respectively (data replicated from Csicsvari et al., 1999). $C$, Theta-related discharge probability of MSDB neurons that were significantly suppressed during SPWs. $D$, Theta phase discharge of MSDB neurons that were not significantly suppressed during SPW. Error bars indicate SE. E, Phase histograms of the two type $1 \mathrm{~A}$ cells. Note the $\sim 90^{\circ}$ phase difference compared with the cells shown in $C$.

locked to the hippocampal theta oscillation (Gaztelu and Buño, 1982; Alonso et al., 1987; King et al., 1998).

It has been suggested that the rhythmic activity of the MSDB neurons is causally related to the generation of hippocampal theta activity (Petsche et al., 1962; Apostol and Creutzfeldt, 1974; Mizumori et al., 1989; Stewart and Fox, 1990; Smythe et al., 1992; Lee et al., 1994; Brazhnik and Fox, 1999). On the other hand, the discovery of the reciprocal circuitry between hippocampal interneurons and MSDB cells (Alonso and Köhler, 1982; Köhler et al., 1984; Freund and Antal, 1988; Tóth et al., 1993) suggested that the pattern of MSDB activity can be affected by the hippocampal output. Early studies using direct stimulation of the hippocampal output (fimbria) showed that downstream inputs to the septum can suppress or reset the bursting activity of MSDB neurons (McLennan and Miller, 1974). Previously, it was tacitly assumed that the hippocampal effects were mediated mostly via the lateral septum neurons (McLennan and Miller, 1974, 1976). However, subsequent anatomical studies revealed that the hypothesized projection from the lateral septum to the MSDB is either very weak or nonexistent (Staiger and Nürnberger, 1991; Gulyás et al., 1991; Leranth et al., 1992). Thus, the main stream of communication between the MSDB area and the hippocampus is by way of septally projecting hippocampal interneurons.

SPWs are associated with the maximum discharge of hippocampal pyramidal cells and the majority of interneurons (Csicsvari et al., 1999). As suggested above, the increased activity of the septally projecting interneurons may be responsible for the suppression of MSDB neurons during SPWs. One might expect that increased discharge of hippocampal interneurons should continue to suppress the activity of the same MSDB population during theta oscillation. However, during theta oscillation, both hippocampal interneurons and the SPW-suppressed MSDB population discharged maximally on the same phase of theta waves, i.e., in a positively correlated manner. Assuming that the septally projecting neurons fired in phase with the remaining population of alveus/stratum oriens interneurons, the observed phase relationship indicates that a simple inhibitory mechanism is not sufficient for the explanation of relationship between these respective neuronal groups during theta state. The state-dependent relationship between hippocampal interneurons and MSDB neurons suggests that the same anatomical pathways are used differentially in different behavioral states. One possibility is that the availability of subcortical neurotransmitters determines whether the firing patterns of hippocampal interneurons and MSDB cells are positively or negatively correlated. Another possible scenario is that the magnitude of the excitatory drive on the participating neurons of the GABAergic septohippocampal loop determines the firing relationship between hippocampal interneurons and MSDB cells. MSDB neurons may be influenced by their reciprocal collaterals, by the hippocamposeptal GABAergic projection and, possibly, by local MSDB GABAergic interneurons (Leranth et al., 1992; Tóth et al., 1993). Depending on the exact functional coupling within these multiple inhibitory loops, the firing frequency of the participating neurons may either increase or decrease (Freund and Buzsáki, 1996).

Although the SPW-suppressed group represents a functional entity, their relationship to the anatomical classes of MSDB neurons is not clear. On the basis of theta phase correlations and the relative magnitude of theta phase modulation, the findings of Brazhnik and Fox (1999, their Fig. 5) would suggest that SPWsuppressed neurons correspond to cholinergic cells and the remaining population to GABAergic neurons. Consequently, increased activity of MSDB GABAergic projecting cells would periodically inhibit hippocampal interneurons at theta frequency (Stuart and Fox, 1990; Lee et al., 1994) and vice versa. A prediction of this scenario is that GABAergic MSDB neurons should be less affected by the septally projecting interneurons than the cholinergic ones, in contrast to the anatomical evidence for the strong hippocampal innervation of septal GABAergic cells (Tóth et al., 1993). Another study did not observe a reliable relationship between theta phase and putative anatomical groups in the MSDB (King et al., 1998). The strongly phase-locked discharge of hippocampal interneurons (Ranck, 1973; Buzsáki and Eidelberg, 
1983; Fox et al., 1986; Skaggs et al., 1996; Csicsvari et al., 1999) and the physiological effectiveness of the septally projecting subgroup, as shown here, indicate that the hippocamposeptal projection may be responsible for the phase-locking of nonrhythmic, slower discharging, putative cholinergic neurons (Griffith and Matthews, 1986; Matthews and Lee, 1991; King et al., 1998) and/or assist the timing of GABAergic neurons (X.-J. Wang, personal communication).

\section{REFERENCES}

Alonso A, Köhler C (1982) Evidence for separate projections of hippocampal pyramidal and non-pyramidal neurons to different parts of the septum in the rat brain. Neurosci Lett 31:209-214.

Alonso A, Gaztelu JM, Buño W, García-Austt E (1987) Crosscorrelation analysis of septohippocampal neurons during theta-rhythm. Brain Res 413:135-146.

Andersen P, Bland BH, Myhrer T, Schwartzkroin PA (1979) Septohippocampal pathway necessary for dentate theta production. Brain Res 165:13-22.

Apostol GH, Creutzfeldt OD (1974) Crosscorrelation between the activity of septal units and hippocampal EEG during arousal. Brain Res 67:65-75.

Barrenechea C, Pedemonte M, Nuñez A, García-Austt E (1995) In vivo intracellular recordings of medial septal and diagonal band of Broca neurons: relationships with theta rhythm. Exp Brain Res 103:31-40.

Brazhnik ES, Fox SE (1997) Intracellular recordings from medial septal neurons during hippocampal theta rhythm. Exp Brain Res 114:442-453.

Brazhnik ES, Fox SE (1999) Action potentials and relations to the theta rhythm of medial septal neurons in vivo. Exp Brain Res, in press.

Buzsáki G, Eidelberg E (1983) Phase relations of hippocampal projection cells and interneurons to theta activity in the anesthetized rat. Brain Res 266:334-339.

Buzsáki G, Leung LWS, Vanderwolf CH (1983) Cellular bases of hippocampal EEG in the behaving rat. Brain Res Rev 6:139-171.

Buzsáki G, Ponomareff GL, Bayardo F, Ruiz R, Gage FH (1989) Neuronal activity in the subcortically denervated hippocampus: a chronic model for epilepsy. Neuroscience 28:527-538.

Buzsáki G, Horváth Z, Urioste R, Hetke J, Wise K (1992) Highfrequency network oscillation in the hippocampus. Science 256:1025-1027.

Carpi D, Dragoi G, Benuck M, Buzsáki G (1997) Neuronal activity of lateral septum during hippocampal theta and sharp waves in the behaving rat. Soc Neurosci Abstr 23:484.

Csicsvari J, Hirase H, Czurkó A, Buzsáki G (1998) Reliability and state dependence of pyramidal cell-interneuron synapses in the hippocampus: an ensemble approach in the behaving rat. Neuron 21:179-189.

Csicsvari J, Hirase H, Czurkó A, Mamiya A, Buzsáki G (1999) Oscillatory coupling of hippocampal pyramidal cells and interneurons in the behaving rat. J Neurosci 19:274-287.

Fox SE, Wolfson S, Ranck Jr JB (1986) Hippocampal theta rhythm and the firing of neurons in walking and urethane anesthetized rats. Exp Brain Res 62:495-508.

Freund TF, Antal M (1988) GABA-containing neurons in the septum control inhibitory interneurons in the hippocampus. Nature 336:170-173.

Freund TF, Buzsáki G (1996) Interneurons of the hippocampus. Hippocampus 6:347-470.

Frotscher M, Leranth C (1985) Cholinergic inervation of the rat hippocampus as revealed by choline acetyltransferase immunocytochemistry: a combined light and electron microscopic study. J Comp Neurol 239:237-246.

Gaykema RPA, van der Kuil J, Hersh LB, Luiten PGM (1991) Patterns of direct projections from the hippocampus to the medial septumdiagonal band complex: anterograde tracing with Phaseoulus vulgaris leucoagglutinin combined with immunohistochemistry of choline acetyltransferase. Neuroscience 43:349-369.

Gaztelu JM, Buño W (1982) Septo-hippocampal relationships during EEG theta rhythm. Electroencephalogr Clin Neurophysiol 54:375-387.

Green JD, Arduini AA (1954) Hippocampal electrical activity in arousal. J Neurophysiol 17:533-557.

Griffith WH, Matthews RT (1986) Electrophysiology of AChE-positive neurons in basal forebrain slices. Neurosci Lett 71:169-174.
Gulyás AI, Seress L, Tóth K, Acsády L, Antal M, Freund TF (1991) Septal GABAergic neurons innervate inhibitory interneurons in the hippocampus of the macaque monkey. Neuroscience 41:381-390.

King C, Recce M, O'Keefe J (1998) The rhythmicity of cells of the medial septum/diagonal band of Broca in the awake freely moving rat: relationships with the behaviour and hippocampal theta. Eur J Neurosci 10:464-477.

Kiss J, Patel AJ (1991) Organisation and synaptic interconnections of parvalbumin and calbindin containing neurons in the rat septum. Third IBRO World Congr Neurosci Abstr 323.

Köhler C, Chan-Palay V, Wu J-Y (1984) Septal neurons containing glutamic acid decarboxylase immunoreactivity project to the hippocampal region in the rat brain. Anat Embryol 169:41-44.

Lee MG, Chrobak JJ, Sik A, Wiley RG, Buzsáki G (1994) Hippocampal theta activity following selective lesion of the septal cholinergic system. Neuroscience 62:1033-1047.

Leranth C, Frotscher M (1989) Organization of the septal region in the rat brain: cholinergic-GABAergic interconnections and the termination of hippocampo-septal fibers. J Comp Neurol 289:304-314.

Leranth C, Deller T, Buzsáki G (1992) Intraseptal connections redefined: lack of a lateral septum to medial septum path. Brain Res 583:1-11.

Lewis PR, Shute CCD, Silver A (1967) Confirmation from choline acetylase analyses of a massive cholinergic innervation to the rat hippocampus. J Physiol (Lond) 191:215-224.

Magee JC, Johnston D (1997) A synaptically controlled, associative signal for Hebbian plasticity in hippocampal neurons. Science 275:209-213.

Markram H, Lubke J, Frotscher M, Sakmann B (1997) Regulation of synaptic efficacy by coincidence of postsynaptic APs and EPSPs. Science 275:213-215.

Matthews RT, Lee WL (1991) A comparison of extracellular and intracellular recordings from the medial septum/diagonal band neurons in vitro. Neuroscience 42:451-462.

McLennan H, Miller JJ (1974) The hippocampal control of neuronal discharges in the septum of the rat. J Physiol (Lond) 237:607-624.

McLennan H, Miller JJ (1976) Frequency-related inhibitory mechanisms controlling rhythmical activity in the septal area. J Physiol (Lond) 254:827-841.

Mizumori SJ, McNaughton BL, Barnes CA, Fox KB (1989) Preserved spatial coding in hippocampal CA1 pyramidal cells during reversible suppression of CA3c output: evidence for pattern completion in hippocampus. J Neurosci 9:3915-3928.

Paxinos G, Watson C (1997) The rat brain in stereotaxic coordinates. San Diego: Academic.

Petsche H, Stumpf CH, Gogolak G (1962) The significance of the rabbit's septum as a relay station between the midbrain and the hippocampus. I. The control of hippocampus arousal activity by the septum cells. Electroencephalogr Clin Neurophysiol 14:202-211.

Raisman G (1966) The connexions of the septum. Brain 89:317-348.

Ranck Jr JB (1973) Studies on single neurons in dorsal hippocampal formation and septum in unrestrained rats. I. Behavioral correlates and firing repertoires. Exp Neurol 41:461-531.

Serafin M, Williams S, Khateb A, Fort P, Mühlethaler M (1996) Rhythmic firing of medial septum non-cholinergic neurons. Neuroscience 75:671-675.

Shute CCD, Lewis PR (1963) Cholinesterase-containing systems of the brain of the rat. Nature 199:1160-1164.

Shute CCD, Lewis PR (1966) Electron microscopy of cholinergic terminals and acetylcholinesterase-containing neurones in the hippocampal formation of the rat. Z Zellforsch Mikrosk Anat 69:334-343.

Skaggs WE, McNaughton BL, Wilson MA, Barnes CA (1996) Theta phase precession in the hippocampal neuronal populations and the compression of temporal sequences. Hippocampus 6:149-172.

Smythe JW, Colom LV, Bland BH (1992) The extrinsic modulation of hippocampal theta depends on the coactivation of cholinergic and GABA-ergic medial septal inputs. Neurosci Biobehav Rev 16:289-308.

Staiger JF, Nürnberger F (1989) Pattern of afferents to the lateral septum in the guinea pig. Cell Tissue Res 257:471-490.

Staiger JF, Nürnberger F (1991) The efferent connections of the lateral septum nucleus in the guinea pig: intrinsic connectivity of the septum and projections to other telencephalic areas. Cell Tissue Res 264:415426. 
Stewart M, Fox SE (1989) Firing relations of medial septal neurons to the hippocampal theta rhythm in urethane anesthetized rats. Exp Brain Res 77:507-516.

Stewart M, Fox SE (1990) Do septal neurons pace the hippocampal theta rhythm? Trends Neurosci 13:163-168.

Stumpf Ch, Petsche H, Gogolak G (1962) The significance of the rabbit's septum as a relay station between the midbrain and the hippocampus. II. The differential influence of drugs upon both the septal cell firing pattern and the hippocampus theta activity. Electroencephalogr Clin Neurophysiol 14:212-219.

Swanson LW, Cowan WM (1979) The connections of the septal region in the rat. J Comp Neurol 186:621-656.

Tóth K, Freund TF (1992) Calbindin $\mathrm{D}_{28 \mathrm{k}}$-containing nonpyramidal cells in the rat hippocampus: their immunoreactivity for GABA and projection to the medial septum. Neuroscience 49:793-805.

Tóth K, Borhegyi Z, Freund TF (1993) Postsynaptic targets of GABAergic hippocampal neurons in the medial septum-diagonal band of Broca complex. J Neurosci 13:3712-3724.
Tóth K, Freund TF, Miles R (1997) Disinhibition of rat hippocampal pyramidal cells by GABAergic afferents from the septum. J Physiol (Lond) 500:463-474.

Tsubokawa H, Ross WN (1997) Muscarinic modulation of spike backpropagation in the apical dendrites of hippocampal CA1 pyramidal neurons. J Neurosci 17:5782-5791.

Van der Zee EA, Luiten PGM (1994) Cholinergic and GABAergic neurons in the rat medial septum express muscarinic acetylcholine receptors. Brain Res 652:263-272.

Vertes RP, Kocsis B (1997) Brainstem-diencephalo-septohippocampal systems controlling the theta rhythm of the hippocampus. Neuroscience 81:893-926.

Winson J (1978) Loss of hippocampal theta rhythm results in spatial memory deficit in the rat. Science 210:160-163.

Ylinen A, Bragin A, Nadásdy Z, Jando G, Szabo I, Sik A, Buzsáki G (1995) Sharp wave-associated high frequency oscillation $(200 \mathrm{~Hz})$ in the intact hippocampus: network and intracellular mechanisms. J Neurosci 15:30-46. 